\title{
Cold magnetized quark matter at finite density in a nonlocal chiral quark model
}

\author{
S.A. Ferraris ${ }^{1}$, D. Gómez Dumm ${ }^{213}$, A.G. Grunfeld $d^{\sqrt{112}}$, N.N. Scoccold $\sqrt{12}^{12}$ \\ ${ }^{1}$ Departamento de Física, Comisión Nacional de Energía Atómica, Av. Libertador 8250, (1429) Buenos Aires, Argentina \\ ${ }^{2}$ CONICET, Godoy Cruz 2290, Buenos Aires, Argentina \\ ${ }^{3}$ IFLP, CONICET - Departamento de Física, Facultad de Ciencias Exactas, Universidad Nacional de La Plata, C.C. 67,1900 \\ La Plata, Argentina
}

Received: date / Accepted: date

\begin{abstract}
We study the behavior of two-flavor dense quark matter under the influence of an external magnetic field in the framework of a nonlocal chiral quark model with separable interactions. The nonlocality is incorporated in the model by using a Gaussian form factor. It is found that for low and moderate values of magnetic field there is a decrease of the critical chiral restoration chemical potential $\mu_{c}$, i.e. an inverse magnetic catalysis effect is observed. For larger values of $e B$ the behavior of $\mu_{c}$ becomes more or less flat, depending on the parametrization. Within the considered parametrization range we do not find a significant growth of the critical chemical potential for large magnetic fields, as occurs in the case of the local NJL model.
\end{abstract}

Keywords Nonlocal chiral quark model · Strong magnetic field

\section{Introduction}

The phase structure of strongly interacting matter under the influence of an external and uniform magnetic field plays an important role in several physical scenarios, such as heavy ion collisions [1, cosmology [2] and compact star physics [3]. For this reason, the analysis of the features of the QCD phase diagram in the presence of an external magnetic field at both finite temperature and/or chemical potential has become an issue of great interest in recent years $4,5,6]$. From the theoretical point of view this kind of study requires to deal with QCD in a low energy regime, where quantitative calculations are extraordinarily difficult in view of the strong coupling involved. One way to overcome

${ }^{\mathrm{a}} \mathrm{e}$-mail: ag.grunfeld@gmail.com this problem is by relying on lattice QCD (lQCD) simulations; however, at finite density they present the harmful sign problem. Then, to circumvent this obstacle, effective models come into the picture as a powerful tool to describe the strongly interacting matter phase diagram at finite densities. It is crucial that effective models show consistency with lQCD simulations at vanishing baryonic chemical potential, before performing extrapolations to higher densities. One of the most widely used approaches in this sense is the Nambu JonaLasinio (NJL) model [7,8]. In this nonrenormalizable effective chiral quark model quarks interact through local four-point vertices, leading to a spontaneous chiral symmetry breaking mechanism $9,10,11$. As an improvement over local models, chiral quark models that include nonlocal separable interactions have also been considered 12,13,14,15. Since these approaches can be viewed as nonlocal extensions of the NJL model, here we denote them generically as "nonlocal NJL" (nlNJL) models. It has been shown that nonlocal approaches include several advantages with respect to the local NJL scheme. For example, the inclusion of smooth nonlocal form factors prevents ultraviolet divergences in momentum space, and the nonlocality leads to a momentum dependence in quark propagators that is consistent with LQCD simulations. In addition, nlNJL models provide a satisfactory description of the hadron properties at zero temperature and density. A recent review with the description and applications of nlNJL models to the analysis of the strong interaction matter under extreme conditions can be found in Ref. [16].

For magnetized quark matter, at zero temperature and vanishing baryonic chemical potential, the results of low-energy effective models of QCD as well as those of IQCD calculations indicate that the size of light quarkantiquark condensates should get increased with the 
magnetic field. Thus, the external field appears to favor the breakdown of chiral symmetry, which is usually known as "magnetic catalysis" (MC) 17. On the contrary, close to the chiral restoration temperature, lQCD calculations carried out with realistic quark masses 18 , 19 show that the condensates behave as nonmonotonic functions of $B$, and this leads to a decrease in the transition temperature when the magnetic field is increased. This effect is known as "inverse magnetic catalysis" (IMC). In addition, lQCD calculations predict an entanglement between the chiral restoration and deconfinement critical temperatures [18]. The observation of IMC has become a challenge for effective models 5, 6. Indeed, most naive effective approaches to low energy QCD predict that the chiral transition temperature should grow with $B$, i.e., they do not find IMC. Interestingly, the corresponding studies carried out in the context of nINJL models [20,21] show that the latter are naturally able to describe, at the mean field level, not only the IMC effect but also the entanglement between chiral restoration and deconfinement transition temperatures. Moreover, it is found that the behavior of the mass and decay constant of the $\pi^{0}$ meson as functions of the external magnetic field are also in agreement with 1 QCD results 22,23 .

In the present work we study the behavior of cold strongly interacting matter under a uniform, static magnetic field in the framework of nlNJL models at finite density. Namely, our aim is to extend the previous works [20,21 to the finite density region. This represents a further step towards the analysis, within these models, of the effect of a strong magnetic field on the full phase diagram of strongly interacting matter. It is important to mention here that in the framework of the local NJL model several studies of the behavior of cold magnetized strongly interacting matter at finite chemical potential have been reported in the literature (see e.g. Refs. [24, 25, 26, 27, 28, 29, 30, 31, 32, 33, 34). One common feature found in those analyses is that for not so strong magnetic fields the critical chemical potential for chiral symmetry restoration decreases with increasing values of $B$. This phenomenon has been also dubbed "inverse magnetic catalysis" in Refs. 35, 36., where the same effect has been found in the context of the Sakai-Sugimoto model. To avoid confusion with the IMC effect at finite temperature mentioned above, here it will be referred to as "inverse magnetic catalysis at finite chemical potential" $(\mu \mathrm{IMC})$. It is worth mentioning that the same effect is obtained when one considers quark-quark channels that give rise to two flavour color superconducting quark matter [37, 38, 39, 40. 41.
The paper is organized as follows. In Sec. 2 we describe the theoretical formalism of cold magnetized quark matter within nlNJL models at finite density. In Sec. 3 we show our results for the chiral limit case and for finite current quark masses. Finally, in Sec. 4 we sketch our conclusions.

\section{Theoretical formalism}

We start by quoting the Euclidean action for the nonlocal chiral quark model under consideration. In the case of two light flavors one has

$$
\begin{array}{r}
S_{E}=\int d^{4} x[\bar{\psi}(x)(-i \not \partial+\hat{m}) \psi(x) \\
\left.-\frac{G}{2} j_{a}(x) j_{a}(x)\right],
\end{array}
$$

where $\psi$ stands for the $u, d$ quark field doublet and $\hat{m}=\operatorname{diag}\left(m_{u}, m_{d}\right)$ is the current quark mass matrix. Throughout this article we will work in the isospin limit, thus we assume $m_{u}=m_{d}$. The currents $j_{a}(x)$ are given by

$j_{a}(x)=\int d^{4} z \mathcal{G}(z) \bar{\psi}\left(x+\frac{z}{2}\right) \Gamma_{a} \psi\left(x-\frac{z}{2}\right)$,

where we have defined $\Gamma_{a}=\left(\mathbb{1}, i \gamma_{5} \vec{\tau}\right)$, and $\mathcal{G}(z)$ is a nonlocal form factor that characterizes the effective interaction.

To describe the behaviour of magnetized quark matter, we include in the effective action a coupling to an external electromagnetic gauge field $\mathcal{A}_{\mu}$. For a local theory, this can be done by introducing a covariant derivative in the kinetic term of the action in Eq. (1), i.e. by changing

$\partial_{\mu} \rightarrow D_{\mu} \equiv \partial_{\mu}-i \hat{Q} \mathcal{A}_{\mu}(x)$,

where $\hat{Q}=\operatorname{diag}\left(q_{u}, q_{d}\right)$ is the electromagnetic quark charge operator $\left(q_{u}=2 e / 3, q_{d}=-e / 3\right)$. In the case of the nonlocal model studied here, this replacement has to be supplemented by a contribution arising from the nonlocal currents in Eq. (2). One has [15]

$\psi(x-z / 2) \rightarrow \mathcal{W}(x, x-z / 2) \psi(x-z / 2)$,

where the function $\mathcal{W}(s, t)$ is defined by

$\mathcal{W}(s, t)=P \exp \left[-i \int_{s}^{t} d r_{\mu} \hat{Q} \mathcal{A}_{\mu}(r)\right]$.

Here $P$ stands for path ordering, and $r$ runs over an arbitrary path connecting $s$ with $t$. As it is usually done, the latter is taken to be a straight line 42 . For simplicity, we restrict to the particular case of a constant and homogeneous magnetic field, which is chosen to be orientated along the 3 -axis. To perform the analytical 
calculations we use the Landau gauge, in which one has $\mathcal{A}_{\mu}=B x_{1} \delta_{\mu 2}$. With this gauge choice the function $\mathcal{W}(s, t)$ in Eq. (5) is given by

$\mathcal{W}(s, t)=\exp \left[-\frac{i}{2} \hat{Q} B\left(s_{1}+t_{1}\right)\left(t_{2}-s_{2}\right)\right]$.

To proceed, we perform a standard bosonization of the theory, introducing scalar and pseudoscalar fields $\sigma(x)$ and $\vec{\pi}(x)$, and integrating out the fermion fields. Moreover, we consider the mean field approximation (MFA), assuming that the field $\sigma(x)$ has a nontrivial translational invariant mean field value $\bar{\sigma}$, while the mean field values of pseudoscalar fields are zero. In the presence of the external magnetic field, it is convenient to write the effective action in a basis of Ritus functions 43. Details of this procedure can be found e.g. in Refs. 20, 21].

Since we are interested in the study of dense quark matter, we consider a system at nonzero quark chemical potential $\mu$ ( $\mu=\mu_{B} / 3$, where $\mu_{B}$ is the baryon chemical potential). Then, the grand canonical thermodynamic potential can be obtained from the effective action, including the chemical potential through the replacement $\partial_{4} \rightarrow \partial_{4}-i \mu$ in the kinetic term. In addition, to obtain the appropriate conserved currents, this has to be supplemented by a modification of the nonlocal currents in Eq. (2) 21]. In practice, if the Fourier transform of the nonlocal form factor $\mathcal{G}(z)$ is denoted by $g(p)$, the latter has to be modified by changing $p_{4} \rightarrow p_{4}+i \mu$. In this way, the thermodynamic potential in the MFA is found to be given by

$$
\begin{aligned}
\Omega_{\mu, B}^{\mathrm{MFA}} & =\frac{\bar{\sigma}^{2}}{2 G}-\sum_{f=u, d} \frac{3 B_{f}}{2 \pi} \int \frac{d^{2} p_{\|}}{(2 \pi)^{2}} \\
& \times\left[\ln \left(p_{\|}^{2}+M_{0, p_{\|}}^{s_{f}, f^{2}}\right)+\sum_{k=1}^{\infty} \ln \Delta_{k, p_{\|}}^{f}\right],
\end{aligned}
$$

where

$$
\begin{aligned}
\Delta_{, p_{\|}}^{f}= & \left(2 k B_{f}+p_{\|}^{2}+M_{k, p_{\|}}^{+, f} M_{k, p_{\|}}^{-, f}\right)^{2} \\
& +p_{\|}^{2}\left(M_{k, p_{\|}}^{+, f}-M_{k, p_{\|}}^{-, f}\right)^{2},
\end{aligned}
$$

with

$M_{k, p_{\|}}^{\lambda, f}=\left(1-\delta_{k_{\lambda},-1}\right) m_{c}+\bar{\sigma} g_{k, p_{\|}}^{\lambda, f}$

and

$$
\begin{aligned}
g_{k, p_{\|}}^{\lambda, f}= & \frac{4 \pi}{\left|q_{f} B\right|}(-1)^{k_{\lambda}} \int \frac{d^{2} p_{\perp}}{(2 \pi)^{2}} g\left(p_{\perp}^{2}+p_{\|}^{2}\right) \\
& \times \exp \left(-p_{\perp}^{2} / B_{f}\right) L_{k_{\lambda}}\left(2 p_{\perp}^{2} / B_{f}\right) .
\end{aligned}
$$

Here, we use the definitions $m_{c}=m_{u}=m_{d}, p_{\perp}=$ $\left(p_{1}, p_{2}\right), p_{\|}=\left(p_{3}, p_{4}+i \mu\right)$ and $k_{ \pm}=k-1 / 2 \pm s_{f} / 2$, where $s_{f}=\operatorname{sign}\left(q_{f} B\right)$. In addition, we denote $B_{f}=$ $\left|q_{f} B\right|$, while $L_{m}(x)$ are Laguerre polynomials, with the usual convention $L_{-1}(x)=0$. It can be seen that the functions $M_{k, p_{\|}}^{ \pm, f}$ play the role of constituent quark masses in the presence of the external magnetic field. The index $k$ is a quantum number that labels the so-called Landau energy levels of quark fields.

In general, the expression in Eq. (7) turns out to be divergent. It can be regularized following the prescription in Ref. [4], namely

$$
\Omega_{\mu, B}^{\mathrm{MFA}, \mathrm{reg}}=\Omega_{\mu, B}^{\mathrm{MFA}}-\Omega_{\mu, B}^{\mathrm{free}}+\Omega_{\mu, B}^{\text {free,reg }},
$$

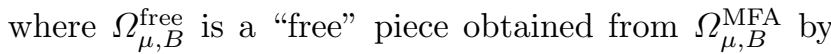
taking $\bar{\sigma}=0$ (and keeping the chemical potential and the interaction with the magnetic field). The regularized form of this free piece is given by

$$
\begin{aligned}
\Omega_{\mu, B}^{\text {free,reg }}= & -\frac{N_{c}}{2 \pi^{2}} \sum_{f=u, d}\left[B_{f}^{2} t_{f}\right. \\
& \left.+\sum_{k} \theta\left(\mu-S_{k f}\right) \alpha_{k} B_{f} v_{k f}\right],
\end{aligned}
$$

where

$t_{f}=\zeta^{\prime}\left(-1, x_{f}\right)+\frac{x_{f}^{2}}{4}-\frac{1}{2}\left(x_{f}^{2}-x_{f}\right) \ln x_{f}$

and

$v_{k f}=\mu \sqrt{\mu^{2}-S_{k f}^{2}}-S_{k f}^{2} \ln \left[\frac{\mu+\sqrt{\mu^{2}-S_{k f}^{2}}}{S_{k f}}\right]$.

Here we denote $x_{f}=m_{c}^{2} /\left(2 B_{f}\right), \alpha_{k}=1-\delta_{0 k} / 2$ and $S_{k f}=\left(m_{c}^{2}+2 k B_{f}\right)^{1 / 2}$. In addition, we use the notation $\zeta^{\prime}\left(z, x_{f}\right)=d \zeta\left(z, x_{f}\right) / d z, \zeta\left(z, x_{f}\right)$ being the Hurwitz zeta function.

Given the regularized form of the mean field thermodynamic potential, the mean field value $\bar{\sigma}$ can be obtained by solving the gap equation

$\frac{\partial \Omega_{\mu, B}^{\mathrm{MFA}, \mathrm{reg}}}{\partial \bar{\sigma}}=0$

In general, for each value of $\mu$ and $B$ several solutions of this equation may exist. The most stable solution will be the one corresponding to the absolute minimum of $\Omega_{\mu, B}^{\mathrm{MFA}, \mathrm{reg}}$. Once $\bar{\sigma}$ has been determined, the chiral quark condensates $\langle\bar{q} q\rangle$, with $q=u, d$, can be calculated from

$\langle\bar{q} q\rangle=\frac{\partial \Omega_{\mu, B}^{\mathrm{MFA}, \mathrm{reg}}}{\partial m_{q}}$. 


\section{Numerical results}

To obtain the numerical predictions that follow from the formalism described in the preceeding section, it is necessary to specify the particular shape of the nonlocal form factor. In the present work we use a Gaussian function

$g\left(p^{2}\right)=\exp \left(-p^{2} / \Lambda^{2}\right)$

where $\Lambda$ is a parameter that indicates the range of the quark level interaction in momentum space. With this particular choice of the form factor, the integral in Eq. 10 can be performed analytically. One gets in this way

$g_{k, p_{\|}}^{\lambda, f}=\frac{\left(1-B_{f} / \Lambda^{2}\right)^{k_{\lambda}}}{\left(1+B_{f} / \Lambda^{2}\right)^{k_{\lambda}+1}} \exp \left(-p_{\|}^{2} / \Lambda^{2}\right)$.

Therefore, the model includes three free parameters, viz. the effective momentum scale $\Lambda$, and the constants $m_{c}$ (current quark mass) and $G$ (coupling constant) that appear in the effective action. In this work we consider two situations. The first one, discussed in Sec. 3.1, corresponds to the chiral limit, in which we fix $m_{c}=0$. Although not really physical, this situation allows for a more clear identification of the possible existing phases and it is, thus, quite useful from a theoretical point of view. The second situation, analyzed in Sec. 3.2, corresponds to the more realistic case in which a finite current quark mass is considered. The way in which the model parameters are determined in each case is discussed in the corresponding subsections.

\subsection{Chiral limit}

In the chiral limit the number of model parameters reduces to only two, viz. $\Lambda$ and $G$. We consider a parametrization in which these are chosen so as to lead to a typical value for the pion decay constant in the chiral limit, $f_{\pi, \mathrm{ch}}=90 \mathrm{MeV}$, and a given value of chiral quark condensate at zero $B$ and $\mu$. To test the sensitivity of our results to the model parameters we consider the cases $-\langle\bar{q} q\rangle_{\mathrm{ch}}^{1 / 3}=230 \mathrm{MeV}$ and $260 \mathrm{MeV}$. The corresponding parametrizations will be denoted as P230ch and P260ch, respectively. For P230ch we find $\Lambda=608.3 \mathrm{MeV}$ and $G \Lambda^{2}=28.43$, while for P260ch the parameter values are found to be $\Lambda=914.6 \mathrm{MeV}$ and $G \Lambda^{2}=17.64$.

Given a set of parameters one can numerically solve the gap equation 15 for different values of the chemical potential and the external magnetic field. As mentioned above, for given values of $\mu$ and $e B$ this equation has in general more than one solution. In the chiral limit considered in this subsection the solution $\bar{\sigma}=0$ is always present, while for a fixed value of $e B$ and low enough values of $\mu$ a second solution with a nonvanishing value $\bar{\sigma}$ also exists. In particular, for $\mu=0$ the latter corresponds to the absolute minimum of the thermodynamic potential, implying that the system lies in a phase in which chiral symmetry is spontaneously broken. Now, if one keeps $e B$ fixed and, starting from $\mu=0$, increases the chemical potential, the value of the thermodynamic potential corresonding to this solution remains unchanged, while that corresponding to the trivial solution $\bar{\sigma}=0$ is found to decrease, approaching the former. At some critical chemical potential $\mu_{c}$ both values coincide, and for $\mu>\mu_{c}$ the trivial solution is the one that corresponds to absolute minimum of $\Omega_{\mu, B}^{\mathrm{MFA}, \mathrm{reg}}$. Thus, at the critical value $\mu=\mu_{c}$, which is in general a function of the magnetic field, the system undergoes a transition to a phase in which chiral symmetry is restored.
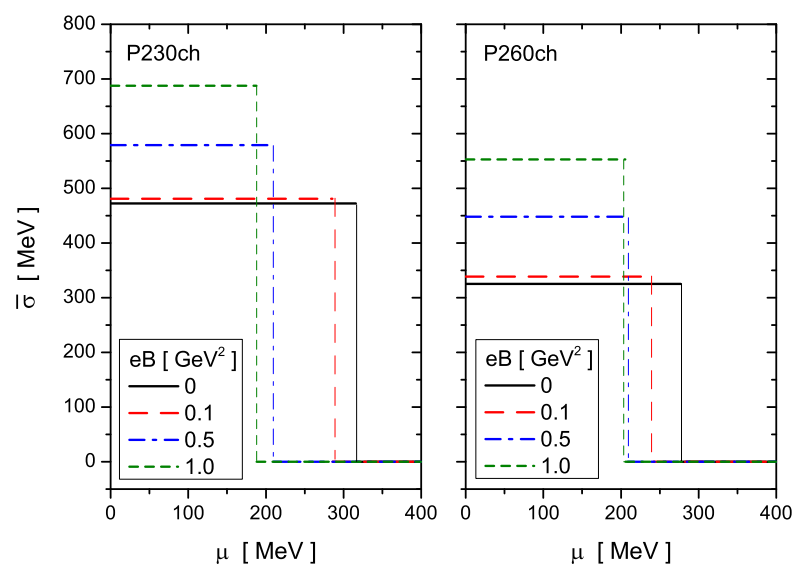

Fig. 1 Mean field value $\bar{\sigma}$ as a function of $\mu$ for some selected values of the $e B$, for parametrizations P230ch (left) and P260ch (right).

The behavior of $\bar{\sigma}$ as a function of $\mu$ for some selected values of the magnetic field, and our two parametrizations, is shown in Fig. 1. The vertical lines correspond to the critical chemical potentials $\mu_{c}$, at which a first order transition is clearly observed. It can be seen that for both parametrizations the value of $\bar{\sigma}$ at $\mu=0$ gets enhanced if $e B$ is increased. This is a manifestation of the well-known "magnetic catalysis" effect, which entails a growth of the absolute value of the condensate with $e B$ in vacuum. In fact, as shown in Refs. [20,21, the rate of this increase is consistent with the results obtained through 1QCD simulations [18,19].

It can also be observed that the parametrization choice has some impact on the values of $\mu_{c}$ as well as on their dependence on the magnetic field. To analyze 
this issue in more detail, in Fig. 2 we plot the value of the critical chemical potential as a function of the parametrization choice, for some selected values of the magnetic field. The parametrization is characterized by
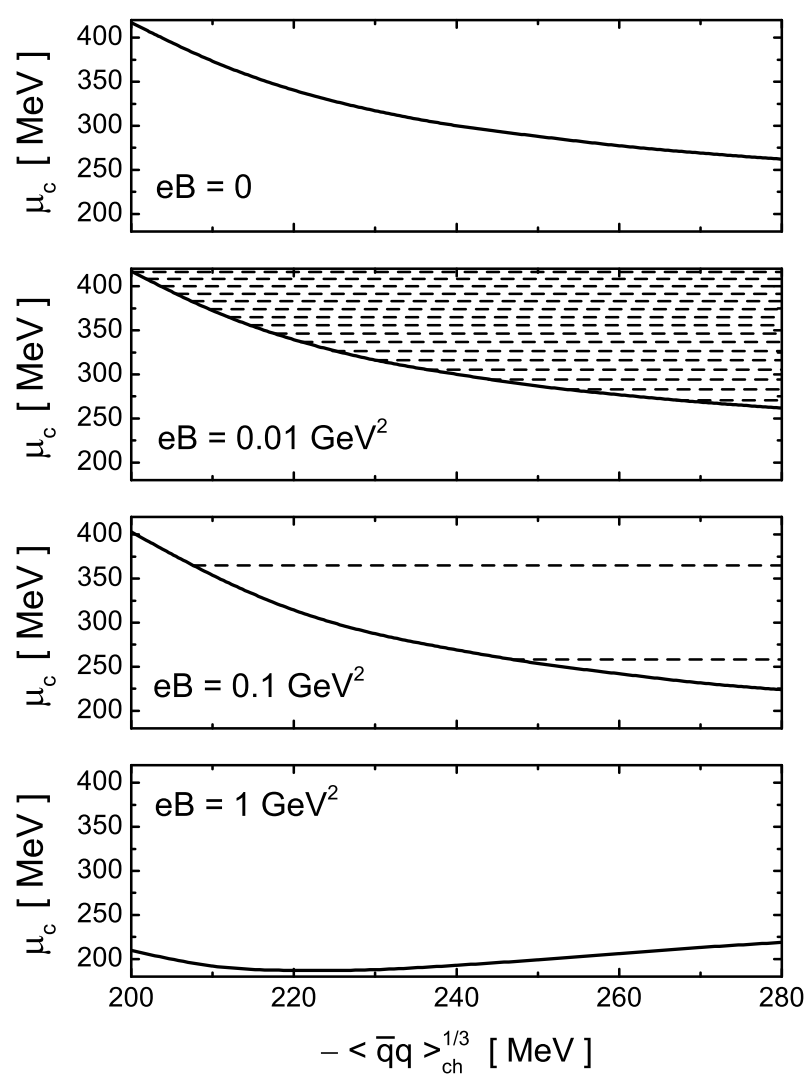

Fig. 2 Critical chemical potential as a function of the parametrization choice, characterized by the value of the quark chiral condensate at zero chemical potential and magnetic field. The graphs correspond to parametrization P230ch and some representative values of $e B$. The dashed lines indicate the chemical potentials corresponding to the vAdH second order phase transitions.

the value of $-\langle\bar{q} q\rangle_{\mathrm{ch}}^{1 / 3}$ in the horizontal axis, which corresponds to a given set of values of $\Lambda$ and $G$ (the remaining input quantity is, as stated, the value of the pion decay constant, $\left.f_{\pi, \mathrm{ch}}=90 \mathrm{MeV}\right)$. Comparing the results obtained for $e B=0$ and $e B=0.01 \mathrm{GeV}^{2}$ (upper panels in Fig. 2 it is seen that although the full line - that corresponds to the first order phase transition between the chiral symmetry broken phase and the chiral symmetry restored phase - is almost identical in both cases, for $e B=0.01$ the $\bar{\sigma}=0$ region is subdivided into many phases. Following the notation in Ref. 24, we denote as phase $\mathbf{B}$ the chiral symmetry broken phase and as $\mathbf{A}_{k}(k=0,1,2, \ldots)$ the chiral symmetry restored phases that show up for finite $e B$.
Each one of the latter corresponds to a different number of populated Landau levels, indicated by the index $k$.

The passage from any of the $\mathbf{A}_{k}$ phases to the next one is known as a van Alphen-de Haas (vAdH) transition. In the chiral limit discussed in this subsection they are regulated by the Heaviside theta function that appears in the last term of Eq. (12). Hence, the transition form the phase $\mathbf{A}_{k-1}$ to the phase $\mathbf{A}_{k}$ happens at a critical chemical potential given by

$\mu_{c, \mathrm{ch}}^{\mathrm{vAdH}}=\sqrt{2 k B_{f}}$.

Clearly, this relation is independent of the parametrization, which explains the fact that in Fig. 2 the dashed lines associated to these transitions are parallel to the horizontal axis. One should keep in mind that in the present case (i.e. for $m_{c}=0$ ) there is no change in the order parameter when one goes from one $\mathbf{A}_{k}$ phase to the next one. In fact, one has $\bar{\sigma}=0$ for all these phases, and, consequently, all these transitions are of second order. The effect of vAdH transitions on physical quantities can be seen, for example, in the quark density $\rho_{q}=-\partial \Omega_{\mu, B}^{\mathrm{MFA}, \mathrm{reg}} / \partial \mu$. This is illustrated in
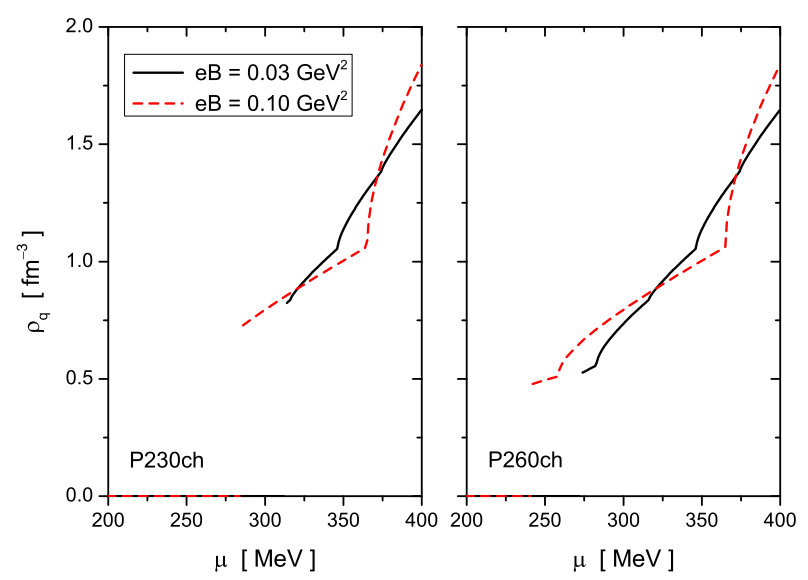

Fig. 3 Quark density as a function of the quark chemical potential, for two representative values of $e B$ and parametrizations P230ch (left) and P260ch (right).

Fig. 3, where we show the behavior of $\rho_{q}$ as a function of $\mu$ for two representative values of the magnetic field and both parametrizations P230ch and P260ch. It can be observed that when the system moves from a given phase $\mathbf{A}_{k}$ to the next one the derivative of the density shows a discontinuity at the transition point.

As it follows from Eq. (19), the number of vAdH transitions in a certain range of chemical potentials depends on the magnetic field strength. This is the reason why in the panel corresponding to $e B=0.01 \mathrm{GeV}^{2}$ one observes a quite large number of vAdH transitions, 
while for $e B=0.1 \mathrm{GeV}^{2}$ there are only two, and none is found for $e B=1 \mathrm{GeV}^{2}$.

Another interesting point to be observed in Fig. 2 is that the dependence of the critical chemical potential for the first order transition with the parametrization (solid lines) is quite similar for $e B=0,0.01 \mathrm{GeV}^{2}$ and $0.1 \mathrm{GeV}^{2}$. In all these cases the value of $\mu_{c}$ decreases as the (absolute) value of the condensate that characterizes the parametrization increases. However, for $e B=$ $1 \mathrm{GeV}^{2}$ the situation is different. For parametrizations corresponding to $-\langle\bar{q} q\rangle_{\mathrm{ch}}^{1 / 3} \gtrsim 220 \mathrm{MeV}$ we find that $\mu_{c}$ slightly increases with the absolute value of the condensate. Thus, one can expect that for large values of $e B$ the behaviour of $\mu_{c}$ would be more sensitive to the chosen parametrization.

In addition, it is important to remark that, for the whole range of parametrizations considered in the present nonlocal model, one finds a direct transition from the phase $\mathbf{B}$ to any of the phases $\mathbf{A}_{k}$. This differs from the situation observed for the case of the local NJL model, in which for some parametrizations one finds intermediate massive phases $\mathbf{C}_{k} 31$.

We conclude this subsection by considering the phase diagrams of magnetized cold quark matter in the $e B-\mu$ plane for parametrizations $\mathrm{P} 230 \mathrm{ch}$ and $\mathrm{P} 260 \mathrm{ch}$, shown in Fig. 4. As stated, $\mathbf{B}$ denotes the spontaneous chiral symmetry broken phase, while $\mathbf{A}_{k}, k=0,1,2, \ldots$, correspond to chiral symmetry restored phases. The second order transitions between $\mathbf{A}_{k}$ phases are indicated by the dashed lines. Each time one of these lines is crossed from right to left, new Landau levels get populated. The crossing from $\mathbf{A}_{0}$ to $\mathbf{A}_{1}$ corresponds to the population of the $d$-quark state with $k=1$, while for the $u$-quark state only the lowest level $k=0$ is allowed. Then, the crossing from $\mathbf{A}_{1}$ to $\mathbf{A}_{2}$ implies the simultaneous population of the $u$-quark state with $k=1$ and the $d$-quark state with $k=2$, etc. The fact that the population of the up quark state with a certain $k$ coincides with the one of the down quark state with $2 k$ is simply due to the fact that (in modulus) the electric charge of the up quark is twice that of the down quark. Regarding the behavior of the first order transition line (solid line in the figure), we can observe three distinct regions. For $e B \lesssim 0.06 \mathrm{GeV}^{2}$ the critical chemical potential $\mu_{c}$ depends only weakly on $e B$, showing a slight decrease as the magnetic field gets increased. Then, in the region from $e B \sim 0.06 \mathrm{GeV}^{2}$ to $e B \sim 0.2 \mathrm{GeV}^{2}$, a much pronounced decrease of $\mu_{c}$ is observed. The behavior found in these two regions is common to both parameterizations in Fig. 4. and it has also been observed in other models like the local NJL model [26,31] and the SakaiSugimoto model 35 . This corresponds to the $\mu \mathrm{IMC}$ effect mentioned in the Introduction. Now, the situation
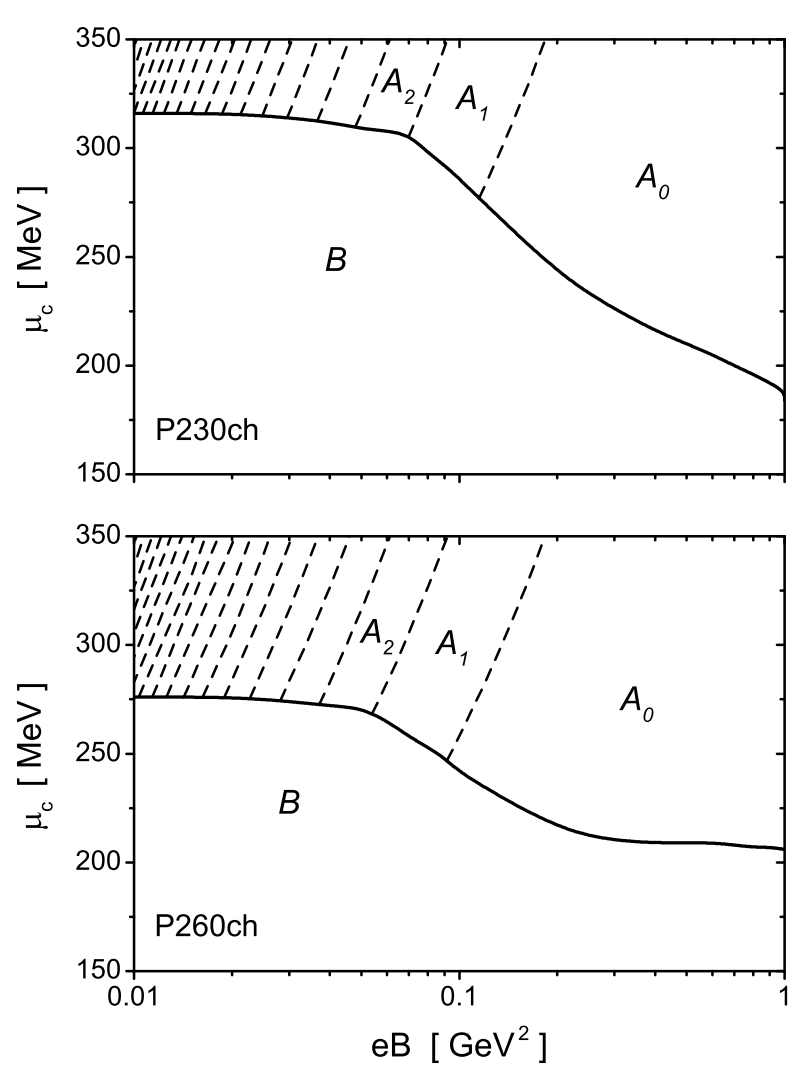

Fig. 4 Phase diagrams in the $e B-\mu$ plane in the chiral limit case, for parametrizations P230ch (up) and P260ch (down). Solid and dashed lines correspond to first and second order phase transitions, respectively.

for magnetic fields larger than $e B \sim 0.2 \mathrm{GeV}^{2}$ turns out to depend on the chosen parametrization, as it has been anticipated from the analysis of Fig. 2. In the case of the parametrization $\mathrm{P} 230 \mathrm{ch}$ it is observed that the decrease of $\mu_{c}$ continues with a rather steep slope, whereas for P260ch it is found that the curve $\mu_{c}(e B)$ becomes almost flat for $e B \gtrsim 0.2 \mathrm{GeV}^{2}$. It should be noted that for no reasonable parametrization of the nonlocal NJL model we find a strong increase of $\mu_{c}$ with $e B$, as it is observed in the local NJL model [31. Actually, we have checked that a qualitatively similar behavior as that found in Fig. 4 is also obtained for other nonlocal form factor shapes, such as Lorenztian-like functions.

\subsection{Finite current quark mass}

In the case of a finite current quark mass, the input parameters of the model are three, namely $\Lambda, G$ and $m_{c}$. They can be set so as to reproduce the phenomenological values of the pion mass and decay constant, $m_{\pi}=139 \mathrm{MeV}$ and $f_{\pi}=92.4 \mathrm{MeV}$, and some acceptable input value of the quark condensate at zero 
$\mu$ and $B$. As in the chiral limit case, we consider the values $-\langle\bar{q} q\rangle^{1 / 3}=230 \mathrm{MeV}$ and $260 \mathrm{MeV}$. The corresponding parametrizations are denoted as P230 and P260, respectively. For P230 one has $\Lambda=677.8 \mathrm{MeV}$, $G \Lambda^{2}=23.65$ and $m_{c}=6.4 \mathrm{MeV}$, whereas for P260 one has $\Lambda=903.4 \mathrm{MeV}, G \Lambda^{2}=17.53$ and $m_{c}=4.6 \mathrm{MeV}$.

As discussed in the previous subsection, once the input parameters have been fixed one can numerically solve the gap equation 15 for given values of the chemical potential and the magnetic field strength. As in the chiral limit case, taking a fixed value of $e B$, for $\mu=0$ the system always lies in a phase $\mathbf{B}$ in which chiral symmetry is spontaneously broken (the absolute minimum of the thermodynamic potential occurs for a solution with a relatively large value of $\bar{\sigma}$ ). Then, if the chemical potential is increased, at some critical value $\mu=\mu_{c}$ the system undergoes a transition to a phase $\mathbf{A}_{k}$, in which $\bar{\sigma}$ jumps to a small value, indicating an approximate restoration of chiral symmetry. This is shown in Fig. 5 . where we quote the phase diagrams in the $e B-\mu$ plane corresponding to parametrizations P230 and P260.
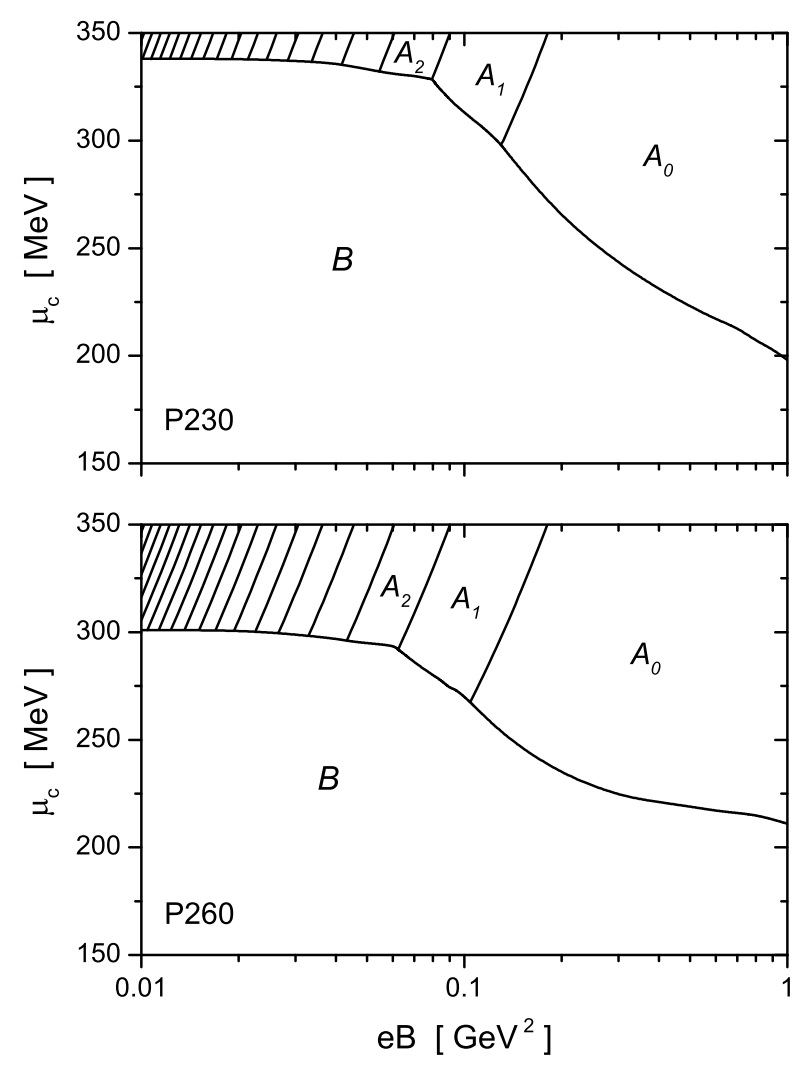

Fig. 5 Phase diagrams for the case of finite current quark masses. Upper and lower panels correspond to parametrizations P230 and P260, respectively.
It can be seen that the phase structure is similar to that found in the chiral limit case. Inverse magnetic catalysis is found for finite $\mu$, as the critical chemical potential decreases for increasing external magnetic field. In addition, it is found that the phase space region in which chiral symmetry is approximately restored is subdivided into many phases $\mathbf{A}_{k}, k=0,1,2, \ldots$, which correspond to different population of the Landau levels. However, notice that in this case the value of the order parameter $\bar{\sigma}$ in the $\mathbf{A}_{k}$ phases is nonvanishing, therefore it can be used to signal not only the chiral restoration transition but also the van Alphen-de Haas transitions. This is illustrated in Fig. 6, where we show the behavior of $\bar{\sigma}$ as a function of $e B$ for parametrization P230, taking three different fixed values of $\mu$. In
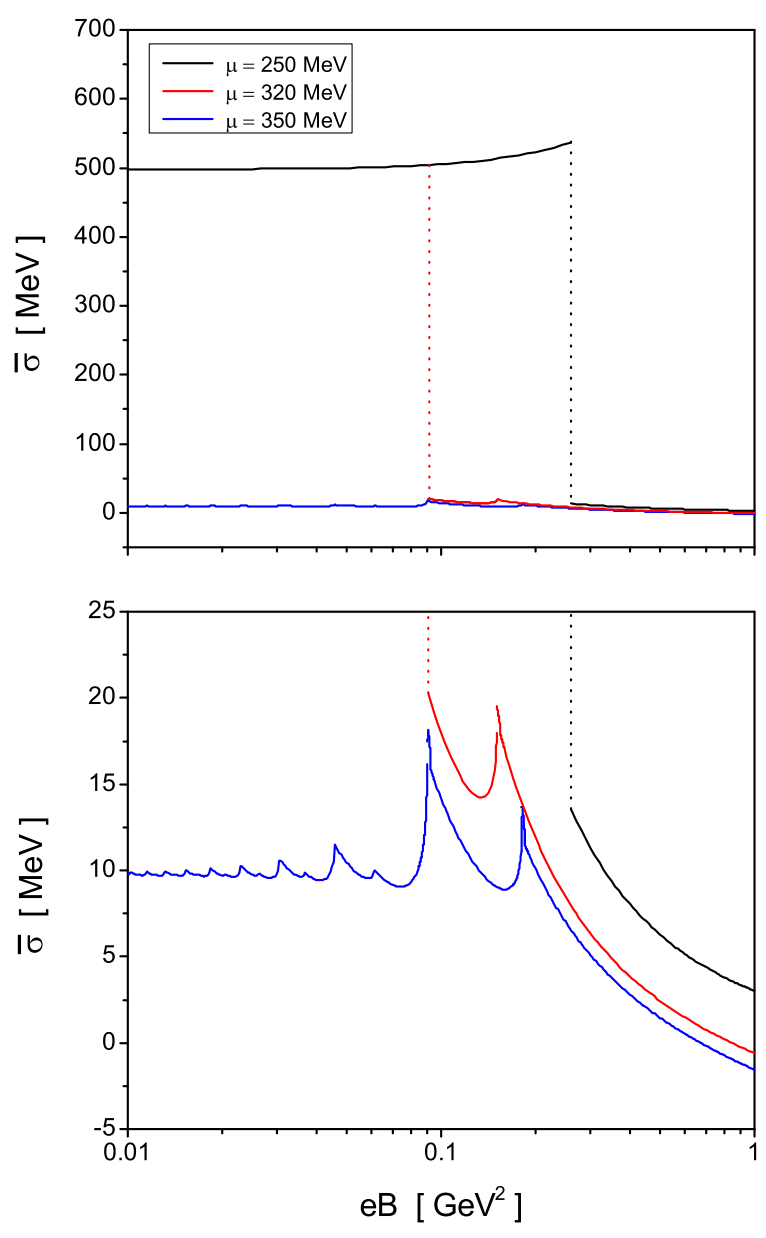

Fig. 6 Mean field value $\bar{\sigma}$ as a function of the magnetic field for three representative values of the quark chemical potential. The results correspond to parametrization P230.

the upper panel, first order chiral restoration transitions are clearly seen for $\mu=250 \mathrm{MeV}$ (black) and $\mu=320 \mathrm{MeV}$ (red), from phase $\mathbf{B}$ to phases $\mathbf{A}_{0}$ and 
$\mathbf{A}_{1}$, respectively. For $\mu=350 \mathrm{MeV}$ the system lies in the chiral restored region for all values of $e B$, therefore the value of $\bar{\sigma}$ remains close to zero (blue). Now, the vAdH transitions can be observed by looking at the lower panel of Fig. 6, in which we plot the same curves as in the upper panel using a different scale and concentrating on the region of small $\bar{\sigma}$. The curve corresponding to $\mu=320 \mathrm{MeV}$ (red) shows, beyond the chiral restoration transition from the $\mathbf{B}$ phase to the $\mathbf{A}_{1}$ phase, a sharp peak denoting a vAdH transition from the $\mathbf{A}_{1}$ phase to the $\mathbf{A}_{0}$ one. A closer look indicates that there is a small discontinuity in the left hand side of this peak, indicating that the $\mathrm{vAdH}$ transition is in fact a first order one. The curve corresponding to $\mu=350 \mathrm{MeV}$ (blue) involves transitions between many $\mathbf{A}_{k}$ states, as can be seen from the phase diagram in Fig. 5 Each transition is characterized by the presence of a peak in $\bar{\sigma}(e B)$, the height of these peaks becoming smaller as the magnetic field gets decreased. As far as numerical calculations can show, these vAdH transition are of first order, therefore they are represented by solid lines in Fig. 5. As discussed for the chiral limit case, the vAdH transitions correspond alternatively to the population of either a new level for the $d$-quark state or a new level for both the $u$ and $d$-quark states. Therefore, they can be grouped into pairs, leading to the pattern observed in the lower panel of Fig. 6. It is worth noticing that the location of the vAdH transition curves in the $e B-\mu$ phase diagram shows just a slight shift with respect to those found in the chiral limit case, given by Eq. (19).

\section{Conclusions}

In the present work we have studied the behavior of cold and dense quark matter in the presence of an external homogeneous magnetic field. We have considered a twoflavor nonlocal NJL model, in which quark-antiquark currents include a Gaussian form factor.

It is found that for low values of the quark chemical potential $\mu$ the system lies in a phase $\mathbf{B}$ in which chiral symmetry is sponteneously broken, while at some critical value $\mu_{c}$ there is a first order phase transition in which this symmetry becomes approximately restored. The restored phase can be subdivided into many phases $\mathbf{A}_{k}$, characterized by the number of populated Landau levels for $u$ and $d$-quark states. One important observation is that for the whole range of parametrizations considered in the present nonlocal model one finds a direct transition from the phase $\mathbf{B}$ to any of the phases $\mathbf{A}_{k}$. This differs from the situation observed for the case of the local NJL model, in which for some parametrizations one finds intermediate massive phases $\mathbf{C}_{k} 31$.
In the chiral limit $m_{c}=0$ it is seen that the van Alphen-de Haas transitions between $\mathbf{A}_{k}$ phases are of second order, and their effect shows up e.g. in the behaviour of the quark density. On the contrary, for finite quark masses these transitions are found to be of first order, though the corresponding jumps in the order parameter $\bar{\sigma}$ are rather tiny. Concerning the first order chiral restoration transition line, it is found that up to $e B \sim 0.2 \mathrm{GeV}^{2}$ the critical chemical potential $\mu_{c}$ decreases with the magnetic field, showing an inverse magnetic catalysis effect. For larger values of $e B$ the behaviour of $\mu_{c}$ becomes more or less flat, depending on the parametrization. In any case, for the considered parametrization range we do not find a significant growth of the critical chemical potential for large magnetic fields, as occurs in the case of the local NJL model.

Acknowledgements This work has been supported in part by Consejo Nacional de Investigaciones Científicas y Técnicas and Agencia Nacional de Promoción Científica y Tecnológica (Argentina), under Grants No. PIP17-700 and No. PICT1703-0571, respectively, and by the National University of La Plata (Argentina), Project No. X824.

\section{References}

1. D. E. Kharzeev, L. D. McLerran and H. J. Warringa, Nucl. Phys. A 803, 227 (2008); V. Skokov, A. Y. Illarionov, and V. Toneev, Int. J. Mod. Phys. A 24, 5925 (2009); V. Voronyuk, V. Toneev, W. Cassing, E. Bratkovskaya, V. Konchakovski, and S. Voloshin, Phys. Rev. C 83, 054911 (2011).

2. T. Vachaspati, Phys. Lett. B265, 258 (1991); K. Enqvist and P. Olesen, Phys. Lett. B319, 178 (1993).

3. R. C. Duncan and C. Thompson, Astrophys. J. 392, L9 (1992); C. Kouveliotou et al., Nature 393, 235 (1998).

4. D. E. Kharzeev, K. Landsteiner, A. Schmitt and H. U. Yee, Lect. Notes Phys. 871, 1 (2013).

5. J. O. Andersen, W. R. Naylor and A. Tranberg, Rev. Mod. Phys. 88, 025001 (2016).

6. V. A. Miransky and I. A. Shovkovy, Phys. Rept. 576, 1 (2015).

7. Y. Nambu and G. Jona-Lasinio, Phys. Rev. 122, 345358 (1961)

8. Y. Nambu and G. Jona-Lasinio, Phys. Rev. 124, 246254 (1961)

9. U. Vogl and W. Weise, Prog. Part. Nucl. Phys. 27, 195$272(1991)$

10. S. P. Klevansky, Rev. Mod. Phys. 64, 649-708 (1992)

11. T. Hatsuda and T. Kunihiro, Phys. Rept. 247, 221-367 (1994) arXiv:hep-ph/9401310 [hep-ph]]

12. S. M. Schmidt, D. Blaschke and Y. L. Kalinovsky, Phys. Rev. C 50, 435-446 (1994)

13. C. J. Burden, L. Qian, C. D. Roberts, P. C. Tandy and M. J. Thomson, Phys. Rev. C 55, 2649-2664 (1997) arXiv:nucl-th/9605027 [nucl-th]]

14. R. D. Bowler and M. C. Birse, Nucl. Phys. A 582, 655664 (1995) arXiv:hep-ph/9407336 [hep-ph]]

15. G. Ripka, "Quarks bound by chiral fields: The quarkstructure of the vacuum and of light mesons and baryons," (Oxford University Press, Oxford, 1997) 
16. D. G. Dumm, J. P. Carlomagno and N. N. Scoccola, Symmetry 13, no.1, 121 (2021) arXiv:2101.09574 [hep$\mathrm{ph}]$.

17. V. P. Gusynin, V. A. Miransky and I. A. Shovkovy, Phys. Rev. Lett. 73, 3499-3502 (1994) [erratum: Phys. Rev. Lett. 76, 1005 (1996)]

18. G. S. Bali, F. Bruckmann, G. Endrodi, Z. Fodor, S. D. Katz, S. Krieg, A. Schafer and K. K. Szabo, JHEP 1202, 044 (2012).

19. G. S. Bali, F. Bruckmann, G. Endrodi, Z. Fodor, S. D. Katz and A. Schafer, Phys. Rev. D 86, 071502 (2012).

20. V. P. Pagura, D. Gomez Dumm, S. Noguera and N. N. Scoccola, Phys. Rev. D 95, 034013 (2017) arXiv:1609.02025 [hep-ph]

21. D. Gomez Dumm, M. F. Izzo Villafañe, S. Noguera, V. P. Pagura and N. N. Scoccola, Phys. Rev. D 96, 114012 (2017) arXiv:1709.04742 [hep-ph]]

22. D. Gomez Dumm, M. F. Izzo Villafañe and N. N. Scoccola, Phys. Rev. D 97, 034025 (2018) arXiv:1710.08950 [hep-ph]]

23. D. Gomez Dumm, M. F. Izzo Villafañe and N. N. Scoccola, Phys. Rev. D 101, 116018 (2020) arXiv:2004.10052 [hep-ph]]

24. D. Ebert, K. G. Klimenko, M. A. Vdovichenko and A. S. Vshivtsev, Phys. Rev. D 61, 025005 (2000) hep$\mathrm{ph} / 9905253$.

25. D. Ebert and K. G. Klimenko, Nucl. Phys. A 728, 203 (2003) hep-ph/0305149.

26. T. Inagaki, D. Kimura and T. Murata, Prog. Theor. Phys. 111, 371 (2004) hep-ph/0312005.

27. D. P. Menezes, M. Benghi Pinto, S. S. Avancini, A. Perez Martinez and C. Providencia, Phys. Rev. C 79, 035807 (2009) arXiv:0811.3361 [nucl-th]].

28. J. K. Boomsma and D. Boer, Phys. Rev. D 81, 074005 (2010) arXiv:0911.2164 [hep-ph]].

29. G. N. Ferrari, A. F. Garcia and M. B. Pinto, Phys. Rev. D 86, 096005 (2012) arXiv:1207.3714 [hep-ph]].

30. E. J. Ferrer and V. de la Incera, Lect. Notes Phys. 871, 399 (2013) arXiv:1208.5179 [nucl-th]].

31. P. G. Allen and N. N. Scoccola, Phys. Rev. D 88, 094005 (2013)

32. S. S. Avancini, D. P. Menezes, M. B. Pinto and C. Providencia, Phys. Rev. D 85, 091901 (2012) arXiv:1202.5641 [hep-ph]].

33. A. G. Grunfeld, D. P. Menezes, M. B. Pinto and N. N. Scoccola, Phys. Rev. D 90, no.4, 044024 (2014) arXiv:1402.4731 [hep-ph]].

34. P. G. Allen, V. P. Pagura and N. N. Scoccola, Phys. Rev. D 91, no.11, 114024 (2015) arXiv:1502.00572 [hep-ph]].

35. F. Preis, A. Rebhan and A. Schmitt, JHEP 1103, 033 (2011) arXiv:1012.4785 [hep-th]];

36. F. Preis, A. Rebhan and A. Schmitt, Lect. Notes Phys. 871, 51 (2013) arXiv:1208.0536 [hep-ph]].

37. S. Fayazbakhsh and N. Sadooghi, Phys. Rev. D 82, 045010 (2010) arXiv:1005.5022 [hep-ph]].

38. T. Mandal and P. Jaikumar, Phys. Rev. C 87, 045208 (2013) arXiv:1209.2432 [nucl-th]].

39. P. G. Allen, A. G. Grunfeld and N. N. Scoccola, Phys. Rev. D 92, no.7, 074041 (2015) arXiv:1508.04724 [hep$\mathrm{ph}]$.

40. T. Mandal and P. Jaikumar, Adv. High Energy Phys. 2017, 6472909 (2017) arXiv:1701.02561 [hep-ph]].

41. M. Coppola, P. Allen, A. G. Grunfeld and N. N. Scoccola, Phys. Rev. D 96, no.5, $056013 \quad$ (2017) arXiv:1707.03795 [hep-ph]].
42. C. Bloch, Kong. Dan. Vid. Sel. Mat. Fys. Med. 27N8, no. 8, 1-55 (1952)

43. V. I. Ritus, Sov. Phys. JETP 48, 788 (1978)

44. D. Gomez Dumm and N. N. Scoccola, Phys. Rev. C 72 , 014909 (2005) arXiv:hep-ph/0410262 [hep-ph]] 